\title{
Semantic Network Array Processor as a Massively Parallel Computing Platform for High Performance and Large-Scale Natural Language Processing*
}

\author{
Hiroaki Kitano \\ Center for Machine Translation \\ Carnegie Mellon University \\ Pittsburgh, PA 15213 U.S.A.
}

\section{Abstract}

This paper demonstrates the utility of the Semantic Network Array Processor (SNAP) as a massively parallel platform for high performance and large-scale natural language processing systems. SNAP is an experimental massively parallel machine which is dedicated to, but not limited to, the natural language processing using semantic networks. In designing the SNAP, we have investigated various natural language processing systems and theories to determine the acope of the hardware support and a set of micro-coded instructions to be provided. As a result, SNAP employs an extended markerpassing model and a dynamically modifiable network model. A set of primitive inatructions is micro-coded to directly support a parallel marker-passing, bitoperations, numeric operations, network modifications, and other essential functions for natural language processing. This paper demonstrates the utility of SNAP for various paradigms of natural language processing. We have discovered that the SNAP provides milliseconds or microseconds performance on several important ap plications such as the memory-based parsing and translation, classification-based parsing, and VLKB search. Also, we ar gue that there are numerous opportunitieg in the NLP community to take advantages of the computational power of the SNAP.

\section{Introduction}

In order to accomplish the high-performance natural language processing, we have designed a highly parallel machine called Semantic Network Array Processor (SNAP) [Lee and Moldovan, 1990]. The goal of our project is to develop and test the validity of the massively parallel machine for high performance and larg-gcale natural language processing. Thus, the architecture of the SNAP was determined reflecting extensive analysis of basic operations essential to the

"This research is supported by the National Science Foundation under grant MIP-9009111 and MIP-9009109, and conducted as a part of IMPACT (International Consortium for Massively Parallel Advanced Computing Technologies)
Dan Moldovan

Department of Electrical Engineering Systems

University of Southern California

Los Angeles, CA 90089-1115 U.S.A.

natural language processing. As a result of the investigation, we have decided to employ an extended marker-passing model and a dymamically modifiable network. Also, a set of primitive instructions is microcoded to directly support essential operations in natural language systems.

Several approach can be taken to use SNAP as a platform for natural language processing systems. We can fully implement NI.P system on SNAP, or we can speed up existing systems by implementing computationally expensive part on SNAP. We have impleinented some of these approaches on SNAP, and obtained extremely high performance (order of milliseconds for given tasks).

In this paper, we describe the design philosophy and architecture of SNAP, and present several approaches toward high performance natural language processing systems on SNAP.

\section{SNAP Architecture}

\subsection{Desigu Philosophy of SNAP}

The Semantic Network Array Processor (SNAP) is a highly parallel array processor fully optimized for semantic network processing with a marker-passing mechanism. The fundermental degign decisions are (1) a semantic network as a knowledge representation scheme, and (2) parallel tnarker-passing as an inference mechanism.

First, the use of a semantic network as a representation scheme can be justified from the fact that most of the representation schemes of current AI and NLP' theories (buch as frame, feature structure, sort hierarchy, systemic choice network, neural network, etc.) can be inspped onto semantic networks. Also, there are numbers of systems and models which directly use semantic networks [Sowa, 1991].

Second, the use of marker-passing can be justified fron several aspects. Obviously, there are many AI and NLP models which use some form of marker-passing as the central computing principle. For example, there are significant number of research being done on word-sense disambiguation as seens in [Waltz and Pollack, 1985], [Hendler, 1988], [Hirst, 1986], [Charniak, 1983], [Tomabechi, 1987], etc. All of them assume passing of markers or values among nodes interconnected via some types of links. There are studies to handle syntactic con- 


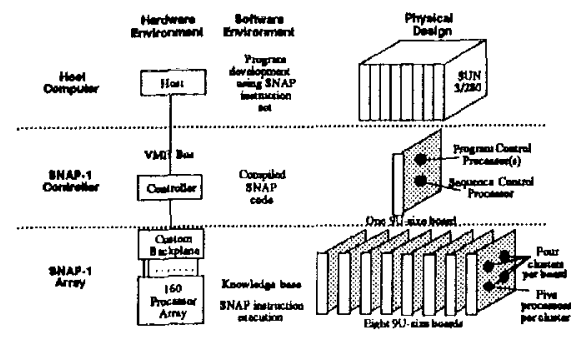

Figure 1: SNAP-1 Architecture

straints using some type of networks which can be mapped onto semantic networks. Recent studies on the Classification-Based Parsing [Kasper, 1989] and the Systemic Choice Network [Carpenter and Pollard, 1991] assume hierarchical networks to represent various linguistic constraints, and the search on these networks can be done by marker-passing. Also, there are more radical approaches to implement entire natural language systems using parallel marker-passing as seen in [Norvig, 1986], [Riesbeck and Martin, 1985], [Tomabechi, 1987], and [Kitano, 1991]. There are, however, differences in types of information carried in each marker-passing model. We will describe our design decisions later.

As reported in [Evett, et. al., 1990], however, serial machines are not suitable for such processing because it causes performance degradation as a size of semantic network increases. There are clear needs for highly parallel machines. The rest of this section provides a brief overview of the SNAP architecture.

\subsection{The Architecture}

SNAP consista of a processor array and an array controller (Figure 1). The processor array has processing cells which contain the nodes and links of a semantic network. The SNAP array consists of 160 processing elements each of which consists of a TMS320C30 DSP chip, local SRAM, etc. Each processing elements atores 1024 nodes which act as virtual processors. They are interconnected via a modified hypercube network. The SNAP controller interfaces the SNAP array with a SUN 3/280 host and broadcasts instructions to control the operation of the array. The instructions for the array are distributed through a global bus by the controller. Propagation of markers and the execution of other instructions can be processed simultaneously.

\subsection{Parallel Marker-Passing}

In the SNAP, content of the marker are: (1) bitvector, (2) address, and (3) numeric value (integer or floating point). In SNAP, the size of the marker is fixed. According to the classification in [Blelloch, 1986], our model is a kind of Finite Message Passing. There are types of marker-, or message-, passing that propagates feature structures (or graphs), which are called Unbounded Message Passing. Although we have extended our marker-passing model from the traditiona] bit marker-passing to the complex marker- passing which carries bits, address, and numeric valnes, we decided not to carry unbounded messages. This is because propagation of feature structures and heavy symbolic operations at each $\mathrm{PE}$ are not practical assumptions to make, at least, on current massively parallel machines due to processor power, mentory capacity on each PE, and the communication bottleneck. Propagation of feature structures would impose serious hardware design problems since the size of the message is unbounded, which means that the designer can not be sure if the local memory size is sufficient or not until the machine actually runs some applications. Also, PEs capable of performing operations to manipulate these messages (such as unification) would be large in physical size which causes assembly problems when thousands of processors are to be assembled into one machine. Since we decide not to support unbounded message passing, we decide to support functionalities attained by the unbounded message passing by other means such as sophisticated marker control rules, dynamic network modifications, etc.

\subsection{Instruction Sets}

A set of 30 high-level instructions specific to semantic network processing are implemented directly in hardware. These include associative search, marker getting and propagation, logical/arithmetic operations involving markers, create and delete nodes and relations, and collect a list of nodes with a certain marker set. Currently, the instruction set can be called from $C$ language so that users can develop applications with an extended version of $\mathrm{C}$ language. From the programming level, SNAP provides dataparallel programming environment similar to $\mathrm{C}^{*}$ of the Connection Machine [Thinking Machines Corp., 1989], but specialized for semantic network processing with marker passing.

Particularly important is the marker propagation rules. Several marker propsgation rules are provided to govern the movement of markers. Marker propagation rules enables us to implement guided, or constraint, marker passing as well as unguided marker passing. This is done by specifying the type of links that markers can propagate. The following are some of the propagation rules of SNAP:

- $S e q(r 1, r 2):$ The $S e q$ (sequence) propagation rule allows the marker to propagate through $r 1$ once then to $r 2$

- Spread $(r 1, r 2)$ : The Spread propagation rule allows the marker to travel through a chain of $r 1$ links and then $r^{2}$ links.

- $C o m b(r 1, r 2)$ : The $C o m b$ (combine) propagation rule allows the marker to propagate to all $r 1$ and r2 links without limitation.

\subsection{Knowledge Representation on SNAP}

SNAP provides four knowledge representation elements: node, link, node color and link value. These elements offer a wide range of knowledge representation schemes to be mapped on SNAP. On SNAP, a concept is represented by a node. A relation can be represented by either a node called relation node or 
a link between two nodes. The node color indicates the type of node. For example, when representing USC is in Los Angeles and CHO is in Pittsburgh, we may assign a relation node for IN. The IN node $i_{8}$ shared by the two facts. In order to prevent the wrong interpretations such as USC in Pittsbargh and CMII in Los Angeles, we assign IN \#1 and IN \#2 to two distinct IN relations, and group the two relation nodes by a node color IN. Wach link has assigned to it a link value which indicates the strength of interconcepts relations. This link value supports probabilistic redsoning and connectionist-like processing. These four basic elements allow SNAP to support virtually any kind of graph-based knowledge representation formalisms such as KL-ONE [Brachman and Schmolze, 1985], Conceptual Graphs [Sowa, 1984], KODIAK [Wilensky, 1987], etc.

\section{The Memory-Based Natural Language Processing}

Memory-based NLP is an idea of viewing NLP as a memory activity. For example, parsing is considered as a memory-search process which identifies similar cases in the past from the memory, and to provide interpretation based on the identified case. It can be considered as an application of Memory-Based Reasoning (MBR) [Stanfill and Waltz, 1986] and CaseBased Reasoning (CBR) [Riesbeck and Scliank, 1989] to NIP. This view, however, connters to traditional idea to view NLP as an extensive rule application process to build up meaning representation. Some models has been proposed in this direction, such as Direct Memory Access Parsing (DMAP) [Riesbeck and Martin, 1985] and $\mathbf{\Phi D M D i a l o g}[$ Kitano, 1991]. For arguments concerning superiority of the memory-based approach over the traditional approach, see [ $\mathrm{NagaO}_{\text {, }}$ 1984], [Riesbeck and Martiu, 1985], and [Sumita and Iida, 1991].

DMSNAP is a SNAP implementation of the ФDMDialog speech-to-speech dialogue translation system which is based on, in part, the menory-based approach. Naturally, it inherits basic ideas and mechanisms of the DMDIALOG system such as a memorybused approach to natural language processing and parallel marker-passing. Syntactic constraint network is introduced in DMSNAP whereas TDMDiaLog has been assuming unification operation to handle linguistic processing.

DMSNAP consists of the memory network, syntactic constraint network, and markers to carry out inference. The memory network and the syntactic constraint network are compiled from a set of grammar rules written for DMSNAP.

Memory Network on SNAP The major types of knowledge required for language translation in DMSNAP are: a lexicon, a concept type hierarchy, concept sequences, and syntactic constraints. Among them, the syntactic constraints are represented in the syntactic constraint network, and the rest of the knowledge is represented in the memory network. The memory network consists of various types of nodes such as concept sequence class (CSC), lexical item nodes (LEX), concept nodes (CC) and others. Nodes are connected by a number of different links such as concept abstraction links (ISA), expression links for both source language and target language (ENG and JPN), Fole links (HOLE), constraint links (CONSTHAINT), contextual links (CON'TEXT) and othexs. A part of the memory network is shown in Figure 2.

Markers The processing of natural language on a marker-propagation architecture requires the creation and movement of markers on the memory network. The following types of markers are used: (1) AMarkers indicate activation of nodes. They propagate through ISA links upward, carry a pointer to the source of activation and a cost measure, (2) $\mathrm{P}$. Markers indicate the next possible nodes to be activated. 'They are initially placed on the first element nodes of the CSCB, and move through NEXT link where they collide with A-MArkens at the element nodes, (3) G-Markers indicate activation of nodes in the target language. They carry pointers to the lexical node to be lexicalized, and propagate through ISA links upward, (4) V-Markers indicate current state of the verbalization. When a V-MArKen collides with the G-MARKER, the surface string (which is specified by the pointer in the G-MARKER) is verbalized, (5) C-Markers indicate contextual priming. Nodes with C-MARKers are contextually primed. A C-MARKER moves from the designated contextual root node to other contextually relevant nodes through contextual links, and (6) SC-Markers indicate active syntax constraints, and primed and/or inhibited nodes by currently active syntactic constraints, It also carries pointer to specific nodes. There are some other markers used for control process and timing; they are not described liere.

The parsing algorithm is sirnilar to the shift-reduce parser except that our algorithms handle ambiguities, parallel processing of each hypothesis, and top-down predictions of possible next input symbol. The gen eration algorithm implemented on SNAP is a version of the lexically guided bottom-up algorithm which is described in [Kitano, 1990]. Details of the algorithm is described in [Kitano et. al., 1991b].

DmSNAP can handle yarious linguistic phenomenn such as: lexical ambiguity, structural ambiguity, referencing (pronoun reference, definite nour reference, etc), control, and unbounded dependencies. Linguistically complex phenomena are handled using the syntactic constraint network (SCN). The SCN enables the DmSNAP to process sentences involving unbounded dependencies, controls without passing feature structures. Jetails of the SCN is described in [Kitano et. al., 1991b]. One notable feature of DmSNAP is its capability to parse and translate sentences in context. In other words, DmSNAP can store results of previous sentences and resolve various levels of ambiguities using the contextual information. Examples of sentences which DmSNAP can handle is shown below. It should be noted that each example consiats of a set of sentences (not a single sentence isolated from the contexi) in order to demonstrate the contextual processing capability of the DMSNAP. 


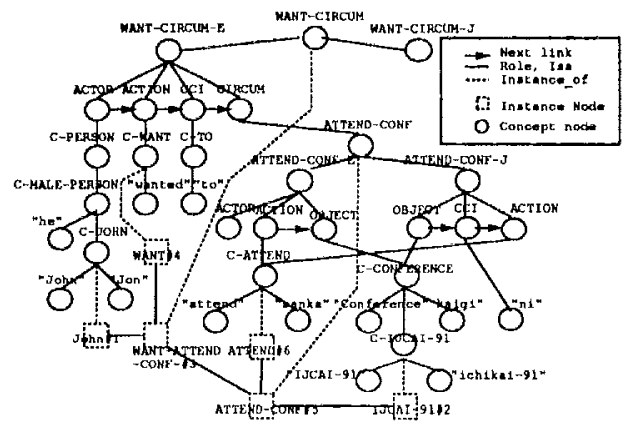

Figure 2: Part of Memory Network

\begin{tabular}{|l|r|r|}
\hline Sentence & $\begin{array}{r}\text { Length } \\
\text { (words) }\end{array}$ & $\begin{array}{r}\text { Time at } \\
10 \mathrm{MHz} \text { (msec) }\end{array}$ \\
\hline s2: He is at ... & 4 & 0.65 \\
\hline s3: He said that ... & 10 & 1.50 \\
\hline s5: Eric build ... & 5 & 0.55 \\
\hline s6: Juntae found ... & 6 & 1.05 \\
\hline s8: Juntae solved ... & 7 & 1.65 \\
\hline
\end{tabular}

Table 1: Execution times for DmSNAP

\begin{tabular}{|ll|}
\hline Example I \\
\hline s1 & John wanted to attend Coling-92. \\
s2 & He is at the conference. \\
s3 & He said that the quality of the paper is superb. \\
\hline \multicolumn{2}{|c|}{ Example II } \\
\hline 84 & Dan planned to develop a parallel processing \\
& computer. \\
s5 & Eric built a SNA.P simulator. \\
s6 & Juntae found bugs in the simulator. \\
s7 Dan tried to persuade Eric to help Juntae modify & the simulator. \\
s8 & Juntae solved a problem with the simulator. \\
s9 & It was the bug that Juntae mentioned. \\
\hline
\end{tabular}

These sentences in examples are not all the sentences which DMSNAP can handle. Currently, DMSNAP handles a substantial portion of the ATR conference registration domain (vocabulary 450 words, 329 sentences) and sentences from other corpora.

The following are examples of translation into Japanese generated by the DmSNAP for the first set of sentences ( 81,82 and 83$)$ :

t1 Jon ha koringu-92 ni sanka shitakatta.

t2 Kare ha kaigi ni iru.

t3 Kare ha ronbun no shitsu ga subarashii to itta.

DMSNAP completes the parsing in the order of milliseconds. Table 1 shows parsing time for some of the example sentences.
F3

$$
\begin{aligned}
& \text { gender male } \\
& \text { number singular } \\
& \text { person } \quad 3 r d
\end{aligned}
$$$$
\text { | gender male }|\quad| \begin{array}{ll}
\text { number } & \text { singular } \\
\text { person } & 3 r d
\end{array} \mid
$$

Figure 3: A part of a simple example of classification lattice

\section{Classiffication-Based Parsing}

Classification-Based Parsing is a new parsing model proposed in [Kasper, 1989]. In the classification-based parsing, feature structures are indexed in the hierarchical network, and an unifiability of two feature structures are tested by searching the Most Specific Subsumer (MSS). The unification, a computationally expensive operation which is the computational bottleneck of many parsing systerns, is replaced by search in the lattice of pre-indexed feature structures.

For example, in Figure 3 , the feature structure F3 is a result of successful unification of the feature structure F1 and F2 (F3 = F1 $\cup$ F2). All feature structures are pre-indexed in a lattice so that the unification is replaced by an intersection search in the lattice with complex indexing. To carry out a search, first we set distinct markers on each feature structures $F 1$ and F2. For example, set marker $M 1$ on $F 1$, and M2 on F2. Then, markers M1 and M2 propagate upward in the lattice. M1 and M2 first co-exist at F3. The most simple program (without disjunctions and conjunctions handling) for this operation follows:

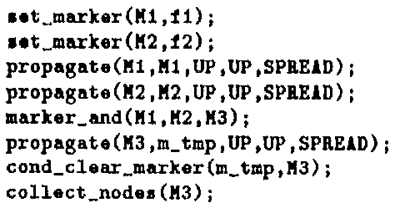

Of course, nodes for each feature structure may need to be searched from a set of features, instead of direct marking. In such a case, a set of markers will be propagated from each node representing each feature, and takes disjunction and conjunction at all nodes representing a feature structure root. This operation can be data-parallel.

There are several motivations to use classificationbased parsing, some of which are described in [Kasper, 1989]. The efficiency consideration is one of the major reasons for using classification-based parsing. Since over $80 \%$ of parsing time has been consumed on unification operations, replacing unification by a faster and functionally equivalent method would substantially benefit the overall performance of the system. The classification-based parsing is efficient because (1) it maximizes structure sharing, (2) it utilizes indexing dependencies, and (3) it avoids redundant computations. However, these advantages of the classification- 

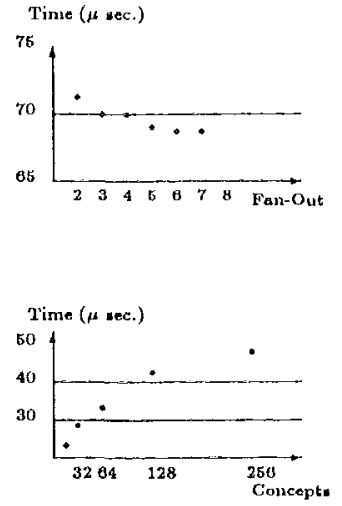

Figure 4: Retrieval Performance on Classification Network

based parsing can not be fully obtained if the model was implemented on the serial machine. This is because a gearch on complex index lattice would be computationally expensive for serial machines. Actually, the time-complexity of the sequential classification algorithm is $O\left(M n^{2}\right)$, and that of the retrieval algorithm is $O\left(R_{\text {ave }} \log M\right)$, where $M$ is a number of concepts, $n$ is an average number of property links per concept, $R_{\text {ave }}$ is an average number of roleset relations for one concept. We can, however, circumvent this problem by using SNAP. Theoretically, time-complexity of the classification on SNAP is $O\left(\log _{F_{o u}} M\right)$, and that of the parallel retrieval is $O\left(F_{\text {in }} D_{\text {ave }}+R_{\text {ave }}\right)$, where $F_{\text {out }}$ is an average fan-out (average number of subconcepts for one concept), $F_{\text {in }}$ is an average fan-in (average number of superconcept for one concept), and $D_{\text {ave }}$ is an average depth of the concept hierarchy [Kim and Moldovan, 1990].

In our model, possible feature structures are precomputed and indexed using our classification algorithrns. While a large set of feature structures need to be stored and indexed, SNAP provides sufficiently large memory/processor space to load an entire feature structure lattice. It is analogous to the idea behind the memory-based parsing which pre-expand all possible syntactic/semantic structures. Here again, we see the conversion of time-complexity into spacecomplexity.

Figure 4 shows performance of retrieval of classification lattice with varying fan-out and size. The clock cycle is $10 \mathrm{MHz}$. It demonstrates that we can attain micro-seconds response for each search. Given the fact that the fastest unification algorithm, even on the parallel machines, takes over few milliseconds per unification, the performance obtained in our experiment promises a significant improvement in parsing apeed for many of the unification-based parsers by replacing unification by classification-based approach.

\section{VLBK Search: Integration with the Knowledge-Based Machine Translation}

Language processing is a knowledge-intensive process. Knowledge-Based Machine Translation (KBMT)[Goodman and Nirenberg, 1991] has been proposed and developed based on the assumption that intensive use of linguistic and world knowledge would provide high quality automatic translation.

One of the central knowledge sources of the KBMT is the ontological hierarchy which encodes abstraction hierarchies of concepts in the given domain, property information of each concept, etc. When a parser creates ambiguous parses or when some parts of the meaning representation (as represented in an interlingua) are missing, this knowledge source is accessed to disambiguate or to fill-in missing information.

However, as the size of the domain scales up, access time to the knowledge source grows to the extent that cost-effective bulk processing would not be possible. For exmaple, [Evett, et. al., 1990] reports that access to large frame systems on serial computers have a time-complexity of $O\left(M \times B^{d}\right)$ where $M$ is the number of conjuncts in the query, $\mathrm{B}$ is the average branching factor in the network, and $d$ is the depth of the network. Thus, even a simplest form of search takes over 6 seconds on a VLKB with $28 K$ nodes measured on a single user node VAX super mini-computer. Since such search on a VLKB must be performed several tirnes for each parse, the performance issue would be a major concern. Considering the fact that VLKB projects such as CYC [Lenat and Guha, 1990] and EDR [EDR, 1988] aim at VLKBs containing over a million concepts, the performance of VLKB search would be an obvious problem in practical use of these VLKBs. In the massively parallel machines such as SNAP, we should be able to attain time-complexity of $O(D+M)$ [Fvett, et. al., 1990].

We have carried out experiments to measure KB access time on SNAP. Figure 5 shows the search time for various size of VIKBs ranging from 800 to $64 \mathrm{~K}$ nodes. Performance was compared with SUN-4 and the CM-2 connection machine. SNAP-1 consistently outperformed other machines (performance curve of SNAP-1 is hard to gee in the figure as it exhibited execution time far less than a second.

\section{Other Approaches}

One clear extension of the currently implemented modules is to integrate the classification-based parsing and the VLKB search. The classification-based parsing carry out high performance syntactic analysis and the VLKB search would impose semantic constraints. Integration of these two would require that the SNAP. 1 to have a multiple controller because two different marker control processes need to be mixed and executed at the same time. Currently SNAP-1 has only one controller. This would be one of the major items for the up-grade of the architecture. However, the performance gain by this appronch would be significant and its impact can be far reaching because a lot of current NLP research has been carried out on the 


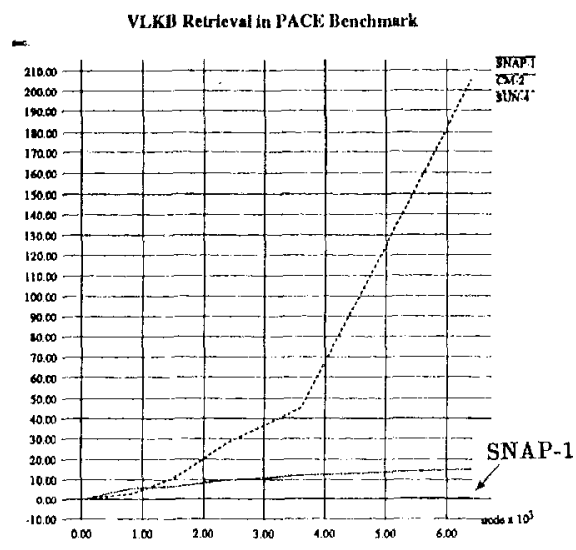

Figure 5: Retrieval time vs. KB size

\section{Conclusion}

In this paper, we have demonstrated that semantic network array processor (SNAP) speeds up various natural language processing tasks. We have demon strated this fact using three examples: the memorybased parsing, VIKB processing, and Classificationbused parsing.

In the memory-based parsing approach, we have attained the speed of parsing in the order of milliseconds without making substantial compromises in linguistic analysis. To the contrary, our model is superior to other traditional natural language processing models in several aspects, particularly, contextual processing.

Next, we have applied the SNAP architecture for a new classification-based parsing model. Here, SNAP is used to search the MSS to test the unifiability of the two feature graphs. We have attained, again, submilliseconds order performance per unifiability test. In addition, this approach exhibited desirable scalability characteristics. The search time asymptotically researches to 450 cycles as the size of classification network increases. Also, search time decreases as average fan-out gets larger. These are natural advantages of using parallel machines.

SNAP is not only useful for the new and radical approach, but also beneficial in speeding up traditional NLP systems such as KBM'T. We have evaluated the performance to search VLKB which is the major knowledge source for the KBMT system. We have attained sub-milliseconds order performance per a search. Traditionally, on the serial machines, this process has been taking a few seconds posing the major thread to performance on the scaled up systems.

Also, there are many other NLP models (Typed Unification Grammar [Emele and Zajac, 1990], SNePS [Neal and Shapiro, 1987], and others) which may exhibit high performance and desirable scaling property on SNAP.

Currently, we are designing the SNAP-2 reflecting various findings made by the research with SNAP-1. SNAP-2 will be built upon the state-of-the-art VLSI technologies using RISC architecture. At least $32 \mathrm{~K}$ virtual nodes will be supported by each processing element, providing the system with a minimum of 16 million nodes. SNAP-2 will feature multi-user supports, intelligent $1 / O$, etc. One of the significant features in SNAP-2 is the introduction of a programmable marker propagation rules. This feature allows users to define their own and more sophisticated marker propagation rules.

In summary, we have shown that the SNAP architecture can be a useful development platform for high performance and large-scale natural language processing. This has been empirically demonstrated using SNAP-1. SNAP-2 is expected to explore opportunities of massively parallel natural language processing.

\section{References}

[Blelloch, 1986] Blelloch, G. E., "CIS; A Massively Parallel Concurrent Rule-Based System," Proceding of 
AAAI-86, 1986.

[Brachman and Schmolre, 1985] Brachion, R. J. and Schmolye, J. G., "An Overview of The KL-ONE Knowledge Representation System," Cognitive Science 9, 171-216, August 1985.

[Charniak, 1983] Charniak, E., "Passing markers: $A$ theory of contextual influence in language comprehengion," Cognitive Science, 7(3), 1983.

[Corpenter and Pollard, 1991] Carpenter, B. and Pollard, C., "Inclusion, Disjointness and Choice: The Logic of Linguistic Classification," Proc. of ACl-91, 1991.

[EDR, 1988] Japan Electric Dictionary Research Institute, EDR Electric Dictionaries, 'Techuical Report, Japan Electric Dictionary Research Institute, 1988.

[Emele and Zajac, 1990] Emele, M. and Zajac, K., "Typed Unification Grammars," Proc. of Coling-90, 1990.

[Fahlinan, 1979] Fahlnan, S., NETL: A Syatem for Representing and Using Real-World Knowledge, The MrT Press, 1979.

[Evett, et. al., 1990] Evett, M., Hendler, J., and Spector, L., PARKA: Parallel Knowledge Representation on the Connection Machine, UMIACS-TR-90-22, University of Maryland, 1990.

[Hendler, 1988] Hendler, J., Integrating Marker-Passing and Problem-Solving, Lawrence Erlbaun Associates, 1988 .

[Hirst, 1986] Hirst, G., Semantic Interpretation and the Resolution of Ambiguity, Cambridge University Press, Cambridge, 1986.

[Jacobs, 1991] Jacobs, P., "Integrating Language and Meaning," Sowa, J. (Ed.) Principles of Semantic Networks, Morgan Kaufmann, 1991.

[Kasper, 1989] Kasper, R., "Unification and Classification: An Experiment in Information-Based Parsing, Proceedings of the International Workshop on Parsing Technologies, Pittaburgh, 1989.

[Kim and Moldovan, 1990] Kim, J. and Moldovan, D., "Parallel Classification for Knowledge Representation on SNAP ${ }^{n}$ Proceedings of the 1990 International Conference on Parallel Processizin, 1990.

[Kitano, 1991] Kitano, H., "\$DmDialog: An Experimental Speech-to-Speech Dialogue Translation System," IEEE Computer, June, 1991.

[Kitano and Higucli, 1991a] Kitano, H. and Higuchi, T, "Massively Parallel Memory-Based Parsing", Proceedings of IJCA1-91, 1991.

[Kitano and Higuchi, 1991b] Kitano, HL. and Higuchi, T., "High Performance Memory-Based Translation on IXM2 Massively Parallel Associative Memory Processor", Proceedings of AAAI-91, 1991.

[Kitano et. al., 1991a] Kitano, H., Hendler, J., Higuchi, T., Moldovan, D., and Waltz, D., "Massively Parallel Artificial Intelligence," Proc. of IJ CA 1-91, 1991.

[Kitano et. al., 1991b] Kitano, H., Moldovan, D., and Cha, S., "High Performance Natural Language Processing on Semantic Network Array Processor," Proc. of IJCAI-91, 1991.

[Kitano, 1990] Kitano, H., "Parallel Incremental Sentence Production for a Model of Simultaneous Interpretation," Dale, R., Mellish, C., and Zock, M. (Eds.) Current Research in Natural Language Generation, Academic Press, London, 1090.

[Kitano et, al., 1989] Kitano, H., Tomabechi, $H_{.1}$ and Levin, I., "Ambiguity Resolution in Dm'Trans Plus," Proceedings of the European Chapter of the Association of Computational Linguiatics, 1989.

[Lee and Moldovan, 1990] Lee, W. and Moldovan, D., "The Design of a Marker Passing Architecture for Knowledge Processing", Proceedings of AAAJ-90, 1990.
[I,enat and Guha, 1990] Lenat, D.B. and Guhn, H.V.; Building Large Knowledge-Based Syutems, AddisonWesley, 1990 .

[Nagao, 1984] Nagao, M., "A Framework of a Mechanical Translation between Japanese and English by Aral. ogy Principle," Artificial and Humati Intelligence, Elithorm, $\Lambda$. and Banerji, R. (Eds.), Elseviex Science Publishers, B.V. 1984.

[Neal and Shnpiro, 1987] Neal, J. and Shapiro, S., "Knowledge-Hased Parsing," Bolc, L., (Ed.) Natural Language Parsing Systemo, Springer-Verlag, 1987.

[Goodman and Nirenberg, 1991] Goodinarl, K., and Nirenberg, S., Knowledge-Based Machine Thansiation "roject: A Case Study, Morgan Kaufrizann, 1991.

[Norvig, 1986] Norvig, P., Unified 'I'heory of Inference for Teat Understanding, Ph.D. Thesis, University of Califorria Berkeley, 1986.

[Pollard and Sag, 1987] Pollard, C. and Sag, I., Information-Based Syntax and Semantics, Vol. I: Fundamentals, CSLI Lecture Note Series, Chicago University Prens, 1987.

[Quillian, 1968] Quillian, M. 1R., "Semantic Memory," Se. mantic Information Processing, Minsky, M. (Ed.), 216-270, The MrT press, Cambridge, MA, 1968.

[Riesbeck and Martin, 1985] Riesbeck, C. and Martin, C., "Direct Memory Access Parsing", Yale University Report 354, 1985.

[Riesbeck and Schank, 1989] Hiesbeck, C. and Schank, R., Inside Case-Based Reasonitı, Lawrence Grlbaum Associates, 1989.

[Sowa, 1991] Sowa, J. F. (Ed.), Principles of Semantic Networks, Morgan Kaufmann, 1991.

[Sows, 1984] Sowa, J.l'., Conceptual Structures, Reading, Addison Wesley, 1984.

[Stanfill and Waltr, 1986] Stanfill, C., and Walts, D., "Toward Menory-Based Reasoning," Communication of the $A C M, 1986$.

[Surnita and lida, 1991] Sunita, E., and Iida, Il., "Experiments and Prospects of Example-Based Macline Translation," Procedings of A CL-91, 1991.

[Thinking Machines Corp., 1989] Thinking Machines Corp., Model CM-2 Technical Summary, 'Ilechrical Report TR-89-1, 1989.

[Tomahechi, 1987] 'Lomabechi, II., "Direct Memory Access 'Translation", Proceedings of the IJCAI-87, 1987.

[Waltz and Pollack, 1985] Waltz, D.I, and Pollack, J., "Massively Parallel Parsing: A Strongly Interactive Model of Natural Language Interpretation" Cognitive Science, 9(1): 51-74, 1985 .

[Wileusky, 1987] Wileasky, R., "Some Problems and Proposals for Knowledge Representation", Technical Report UCB/CSD 87/351, University of Califormia, Berkeley, Computer Science Division, 1987.

[Zajac, 1989] Zajac, R., "A Thansfer Model Using a Typed Feature Structure Rewriting System with Inheri. tance," Proc. of A CL-89, 1089. 